\title{
Features and Prognostic Value of Quantitative Electroencephalogram Changes in Critically III and Non-critically III Anti-NMDAR Encephalitis Patients: A Pilot Study
}

\author{
Nan Jiang, Hongzhi Guan, Qiang Lu, Haitao Ren and Bin Peng* \\ Department of Neurology, Peking Union Medical College Hospital, Chinese Academy of Medical Sciences, Beijing, China
}

OPEN ACCESS

Edited by:

Liping Liu,

Beijing Tiantan Hospital, Capital

Medical University, China

Reviewed by:

Teneille Emma Gofton,

University of Western Ontario, Canada

Benjamin Aaron Emanuel, University of Southern California,

United States

*Correspondence:

Bin Peng

pengbin3@hotmail.com

Specialty section

This article was submitted to Neurocritical and Neurohospitalist

Care,

a section of the journal

Frontiers in Neurology

Received: 08 July 2018 Accepted: 18 September 2018 Published: 05 October 2018

Citation:

Jiang N, Guan H, Lu Q, Ren H and

Peng B (2018) Features and Prognostic Value of Quantitative Electroencephalogram Changes in

Critically III and Non-critically III Anti-NMDAR Encephalitis Patients: A

Pilot Study. Front. Neurol. 9:833.

doi: 10.3389/fneur.2018.00833
Anti-N-methyl-D-aspartate receptor (NMDAR) encephalitis is a common cause of encephalitis in intensive care units. Until now, no reliable method has existed for predicting the outcome of anti-NMDAR encephalitis. In this study, we used quantitative electroencephalography (qEEG) to examine the brain function of anti-NMDAR encephalitis patients and assessed its predictive value. Twenty-six patients diagnosed with anti-NMDAR encephalitis were included and grouped according to whether they were treated in intensive care units (14 critically ill vs. 12 non-critically ill). All patients underwent 2-h 10-channel qEEG recordings at the acute stage. Parameters, including amplitude-integrated electroencephalogram (aEEG), spectral edge frequency 95\%, total power, power within different frequency bands $(\delta, \theta, \alpha$, and $\beta)$, and percentages of power in specific frequency bands from frontal and parietal areas were calculated with NicoletOne Software and compared between groups. The short-term outcome was death or moderate/severe disability at 3 months after onset, measured with a modified Rankin Scale, and the long-term outcome was death, disability or relapse at 12 months. No differences in qEEG parameters were observed between the critically ill and non-critically ill patients. However, differential anterior-to-posterior alterations in $\delta$ and $\beta$ absolute band power were observed. Logistic regression analysis revealed that a narrower parietal aEEG bandwidth was associated with favorable long-term outcomes (odds ratio, 37.9; $P=0.044$ ), with an optimal cutoff value of $1.7 \mu \mathrm{V}$ and corresponding sensitivity and specificity of 90.00 and $56.25 \%$, respectively. In a receiver operating characteristic analysis, the area under the curve was 0.7312. In conclusion, the qEEG parameters failed to reflect the clinical severity of anti-NMDAR encephalitis. However, the parietal aEEG bandwidth may separate patients with favorable and poor long-term outcomes in early stages. The underlying mechanisms require further investigation.

Keywords: anti-NMDAR encephalitis, quantitative EEG, amplitude-integrated EEG, prognosis, intensive care unit 


\section{INTRODUCTION}

Anti-N-methyl-D-aspartate receptor encephalitis is an autoimmune encephalitis involving antibodies directed against the NR1 subunit of the NMDA receptor (NMDAR), and is often associated with ovarian teratomas $(1,2)$. A considerable proportion of anti-NMDAR encephalitis patients fall into coma or develop status epilepticus and require intensive care. In one retrospective study, anti-NMDAR encephalitis accounted for $1 \%$ of all admissions of young adults to intensive care units (ICUs) (3).

Early identification of neurological outcomes is important in terms of therapeutic options. Several prognostic measures have been evaluated in anti-NMDAR encephalitis, including the Glasgow Coma Scale (GCS) score, number of complications, catatonia-predominant type, and electroencephalogram (EEG) (4-6). EEG is a commonly used monitoring tool at the bedside in the ICU, and an EEG "extreme delta brush" (EDB) pattern has been described in anti-NMDAR encephalitis and shown to be a marker of more severe disease and perhaps worse outcome (6). However, the identification of EDB on raw EEG requires experienced raters, which is inconvenient for the continuous monitoring of critically ill patients in the ICU. In addition, the prevalence of EDB is $<30 \%$ in anti-NMDAR encephalitis patients; therefore, it may not be a sensitive prognostic factor (6-9). Hence, exploration for additional EEG markers to measure severity and predict the outcomes of anti-NMDAR encephalitis is needed, especially for cases where EDB is absent.

A variety of quantitative EEG (qEEG) parameters have been developed in neurocritical care practice, including some applied to the diagnosis of viral encephalitis (10). By applying fast Fourier transformation or other techniques, EEG can be quantified in terms of amplitude, power, frequency, and rhythmicity to generate numerical values, ratios, or percentages (11). Some qEEG parameters may provide quantitative information about short-term and long-term outcomes (11). In this study, we sought to explore features of brain background activity using quantitative analyses of EEG in anti-NMDAR encephalitis patients. The relationships of qEEG characteristics with disease outcomes were also assessed.

\section{METHODS}

\section{Participant Enrollment, Data Collection, and Follow-Up}

This single-center retrospective observational study was approved by the ethics committee of Peking Union Medical College Hospital. Eligible patients were enrolled from April 2014 to May 2017. Patient consent was not required because de-identified data were used in this study.

All enrolled patients met the diagnostic criteria for antiNMDAR encephalitis introduced in 2016 (12). Exclusion criteria were as follows: (1) age at onset $<12$ years; (2) identifiable intracranial infections, other autoimmune encephalitis, or other etiologies causing admission to the ICU; (3) inability to cooperate with qEEG monitoring, due to issues such as agitation; (4) known medical history of a severe neurological deficit [modified Rankin
Scale ( $m R S), \geq 2$ ] before onset of anti-NMDAR encephalitis; (5) lack of qEEG recording during the first week of hospitalization in our institute; and (6) missing data or loss to follow-up. According to the severity of disease, patients were further divided into critically ill or non-critically ill subgroups. Patients enrolled in the critically ill subgroup satisfied the following criteria: treated in the ICU for at least $48 \mathrm{~h}$ because of (1) GCS $\leq 8$, (2) status epilepticus, or (3) hypoventilation or severe autonomic dysfunction. Patients who did not meet these criteria comprised the non-critically ill subgroup.

We collected the demographic, clinical and laboratory data of patients, including symptoms at the acute stage, serum and CSF studies, and therapeutic regimens. The short-term outcome for this study was death or degrees of disability, which was evaluated with the mRS at 3 months after onset. Patients were considered to have a favorable short-term outcome when their $\mathrm{mRS}$ scores were $\leq 2$ without increasing compared with baseline, and poor short-term outcome if the mRS scores were $\geq 3$ or had increased. Long-term outcomes were obtained from hospital medical records or face-to-face interviews 12 months after onset. Patients with $\mathrm{mRS}$ scores $\geq 3$ for the whole experimental period or experienced relapse events and received another episode of first-line immunotherapy were defined as experiencing poor long-term outcomes. Patients were considered to have favorable long-term outcomes if their mRS scores were $\leq 2$ with no relapse events.

In the final analysis, a total of 26 patients completed 1-year follow-up. Of these, 25 patients were diagnosed with definite anti-NMDAR encephalitis, and 1 patient met the diagnostic criteria for antibody-negative anti-NMDAR encephalitis. We also recruited 10 healthy volunteers with similar ages and collected their qEEG information as a control group.

\section{EEG Recording and Interpretation}

qEEG monitoring was performed for at least $2 \mathrm{~h}$ for each patient during the first week after admission to our center. Medication administrations during EEG recording were noted and intravenous anti-epileptic agents as well as sedatives were suspended before the start of qEEG monitoring. Silver-chloride disc electrodes were placed according to the International 10-20 System, with a 10-channel layout at the Fp1, F3, C3, P3, O1, and Fp2, F4, C4, P4, O2 sites. The reference electrode was located at $\mathrm{Cz}$, and $\mathrm{Fz}$ was used as ground. A 1.0-Hz lowand $35-\mathrm{Hz}$ high-frequency filter was used. Impedances were maintained below $10 \mathrm{k} \Omega$. The qEEG recording was performed using a NicoletOne EEG monitor (VIASYS Healthcare Inc.), and both raw EEG and processed qEEG tracings were sampled simultaneously. The aEEG, spectral edge frequency 95\% (SEF95), total power, power within $\delta, \theta, \alpha$, and $\beta$ frequency bands, as well as relative percentages of power in specific frequency bands were calculated automatically and exported via NicoletOne system.

All qEEG recordings were analyzed off-line. For each patient, we selected one 30-min artifact-free epoch manually from the F3-F4 and P3-P4 montage for further quantitative analysis. We selected F3-F4 as the representative area of the anterior cross-cerebral EEG signal, and P3-P4 as 
the representative area of the posterior cross-cerebral EEG signal. Because signals from Fp1-Fp2 and O1O2 often contain more artifacts or higher impedances, we did not select these recordings for final quantitative analysis.

\section{Statistical Analysis}

Descriptive data are presented as mean \pm standard deviation (SD) or median with interquartile ranges (IQR). We use Student's $t$-test or the Mann-Whitney $U$-test for group comparisons of continuous variables, and Fisher's exact test for categorical variables, as appropriate. The Kruskal-Wallis test with Bonferroni correction was used for multiple comparisons. Wilcoxon signed rank tests were performed to compare brain activity between anterior and posterior regions. Univariate and multivariate logistic regression with stepwise estimation method was performed to find the independent predictive ability of outcome predictors. Receiver operator characteristic (ROC) curves and areas under the curve (AUC) were constructed to study the ability of aEEG to predict outcomes. For these 2tailed tests, $p<0.05$ was considered statistically significant. Statistical analyses were performed using Stata software version 14.1.

\section{RESULTS}

\section{Patient Characteristics}

Of the 26 patients, 11 (42.3\%) were male and 15 (57.7\%) were female. The median age was 20 (IQR: 16-27) years. No tumor was found in any of the male patients. Eight (53\%) female patients had underlying tumors, which were pathologically confirmed as ovarian teratomas. All patients received intravenous immunoglobulin (2 g/kg divided for 5 days), 25 (96.2\%) treated with methylprednisolone (1 g/d for at least 3 days), and $14(53.8 \%)$ patients received second-line immunotherapy. Table 1 summarizes the comparison of demographic and clinical information between critically ill and non-critically ill patients.

\section{Patient Outcomes}

Patient short-term outcomes were as follows: favorable outcome, 7 of 26 (26.9\%); and poor outcome, 19 of 26 (73.1\%). There was no significant difference between the two subgroups in terms of demographic information, CSF profiles, concomitant tumors, or immunotherapy regimens. All patients in the favorable shortterm outcome subgroup had symptoms of impaired memory at admission ( $7 / 7$ vs. $6 / 19, p=0.005)$, and patients with impaired consciousness on admission were more likely to have poor short-term outcomes ( $1 / 7$ vs. $13 / 19, p=0.026)$. Short-term outcomes of the critically ill subgroup were worse than those of the non-critical subgroup (1/7 vs. $13 / 19, p=$ 0.026).

There were $10(38.5 \%)$ patients experiencing poor longterm outcomes, including 5 with $\mathrm{mRS} \geq 3$ and 7 with relapse events within 12 months. One patient died because of complications due to infection. There were no significant differences between the favorable long-term outcome group and poor long-term outcome group in terms of sex, age, clinical
TABLE 1 | Comparison of demographic, clinical, and CSF characteristics between critically ill and non-critically ill patients.

\begin{tabular}{|c|c|c|c|}
\hline & $\begin{array}{l}\text { Critically III } \\
\text { subgroup }\end{array}$ & $\begin{array}{c}\text { Non-critically III } \\
\text { subgroup }\end{array}$ & $P$-value \\
\hline \multicolumn{4}{|l|}{ DEMOGRAPHIC } \\
\hline Gender(female) & $8 / 14$ & $7 / 12$ & 1 \\
\hline Age & $20(15,26)$ & $20.5(17,31)$ & 0.502 \\
\hline \multicolumn{4}{|l|}{ CLINICAL INFORMATION } \\
\hline Fever & $13 / 14$ & $4 / 12$ & $0.003^{*}$ \\
\hline Headache & $10 / 14$ & $4 / 12$ & 0.113 \\
\hline Psychiatric behaviour & $12 / 14$ & $11 / 12$ & 1 \\
\hline Cognition dysfunction & $4 / 14$ & $6 / 12$ & 0.422 \\
\hline Memory impairment & $4 / 14$ & $9 / 12$ & $0.047^{\star}$ \\
\hline Speech dysfunction & $4 / 14$ & $8 / 12$ & 0.113 \\
\hline Seizures & $13 / 14$ & $11 / 12$ & 1 \\
\hline Movement disorder & $9 / 14$ & $6 / 12$ & 0.692 \\
\hline Central hypoventilation & $7 / 14$ & $0 / 12$ & $0.006^{*}$ \\
\hline Autonomic dysfunction & $10 / 14$ & $5 / 12$ & 0.233 \\
\hline Decreased consciousness & $11 / 14$ & $3 / 12$ & $0.016^{*}$ \\
\hline Glasgow Coma Scale & $5(3,6)$ & $11.5(7,15)$ & $0.005^{\star}$ \\
\hline Days till diagnosis & $20(15,25)$ & $17.5(13.5,34.5)$ & 0.959 \\
\hline Days in hospital & $61(54,120)$ & $16.5(13,27.5)$ & $0.0002^{*}$ \\
\hline Mechanical ventilation & $10 / 14$ & $0 / 12$ & $0.0001^{*}$ \\
\hline Tumor & $7 / 14$ & $1 / 12$ & $0.036^{\star}$ \\
\hline Tumor in female & $7 / 8$ & $1 / 7$ & $0.01^{*}$ \\
\hline Elevated CSF protein & $2 / 14$ & $5 / 12$ & 0.19 \\
\hline CSF leukocyte & & & 0.728 \\
\hline$\sim 5$ & $8 / 14$ & $6 / 12$ & \\
\hline $6 \sim 50$ & $5 / 14$ & $5 / 12$ & \\
\hline $51 \sim$ & $1 / 14$ & $1 / 12$ & \\
\hline Oligoclonal band & & & 0.642 \\
\hline Negative & $6 / 13$ & $3 / 10$ & \\
\hline Suspected & $0 / 13$ & $5 / 10$ & \\
\hline Positive & $7 / 13$ & $2 / 10$ & \\
\hline Antibody titers in CSF & & & 0.324 \\
\hline$\sim 1: 10$ & $1 / 14$ & $1 / 12$ & \\
\hline $1: 32$ & $3 / 14$ & $5 / 12$ & \\
\hline $1: 100 \sim$ & $10 / 14$ & $6 / 12$ & \\
\hline Antibody titers in serum & & & $0.040^{*}$ \\
\hline Negative & $3 / 14$ & $7 / 12$ & \\
\hline $1: 10$ & $2 / 14$ & $1 / 12$ & \\
\hline $1: 32 \sim$ & $9 / 14$ & $4 / 12$ & \\
\hline \multicolumn{4}{|c|}{ SECOND-LINE IMMUNOTHERAPY } \\
\hline MMF & $9 / 14$ & $4 / 12$ & 0.238 \\
\hline MTX & $4 / 14$ & $0 / 12$ & 0.100 \\
\hline CTX & $1 / 14$ & $0 / 12$ & 1 \\
\hline RTX & $1 / 14$ & $1 / 12$ & 1 \\
\hline No 2nd-line Immunotherapy & $4 / 14$ & $8 / 12$ & 0.113 \\
\hline
\end{tabular}

symptoms, severity of onset, duration of hospital stay, profiles in CSF, concomitant tumors, or immunotherapy regimens during hospitalization. 


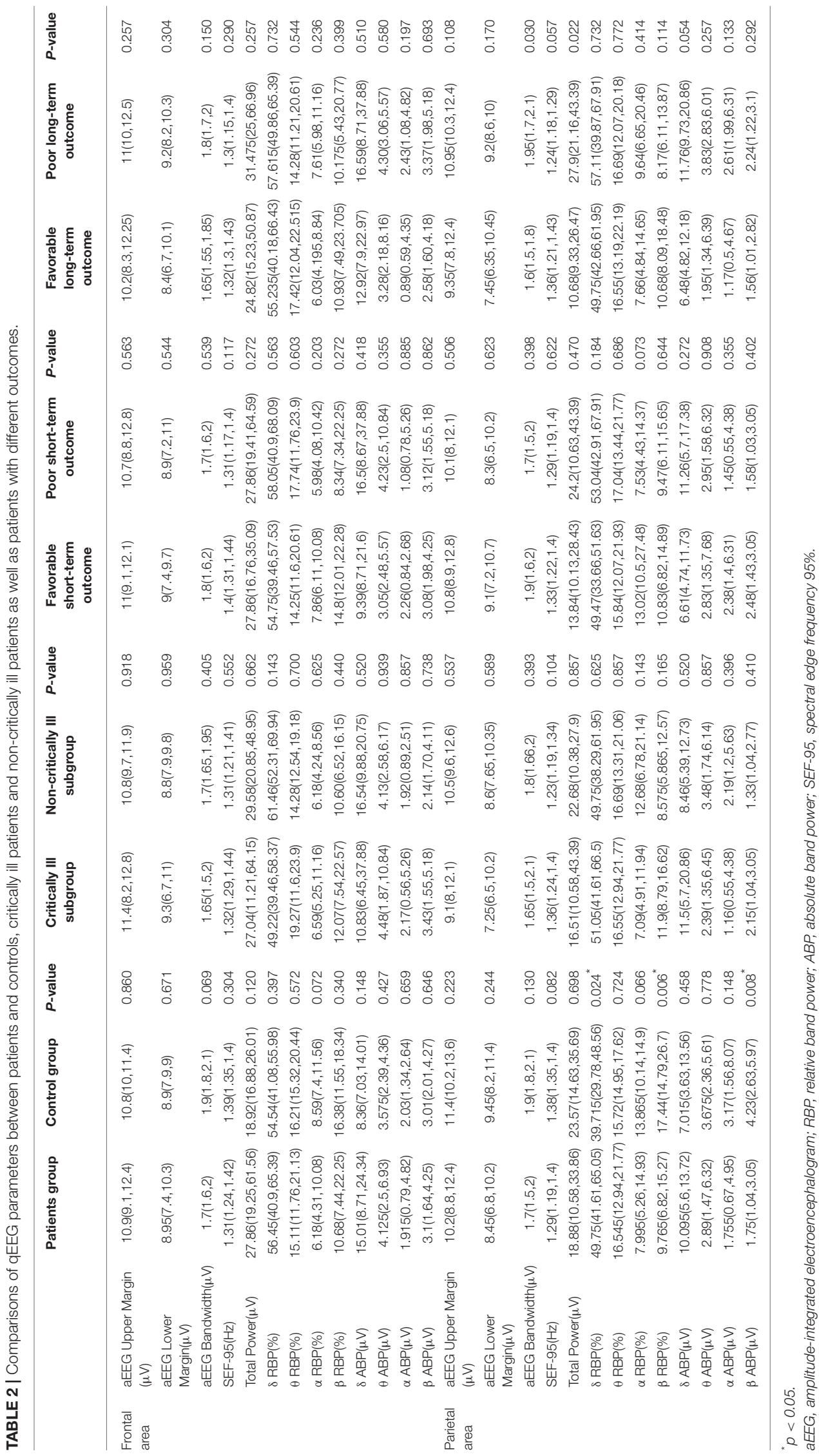




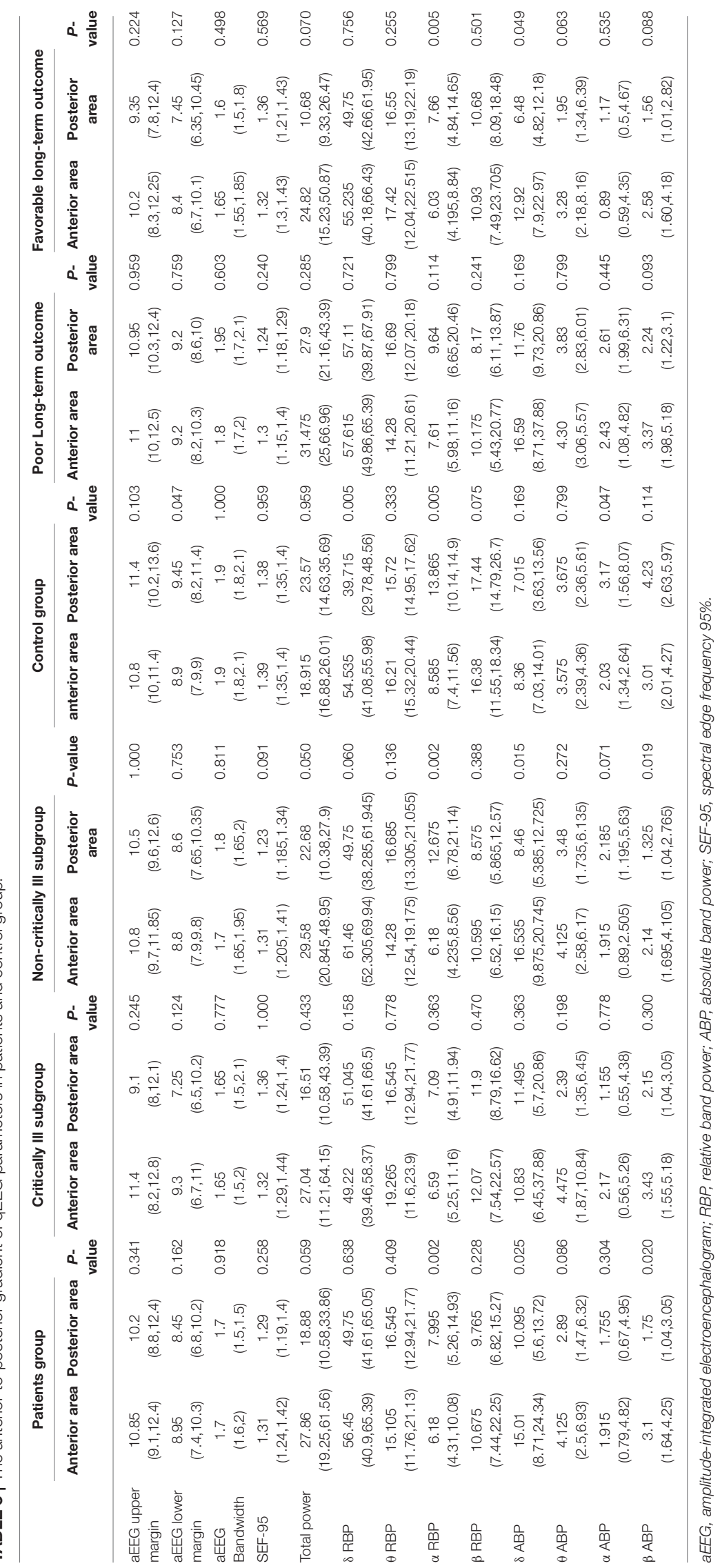




\section{qEEG Findings}

The detailed results for qEEG parameters are presented in Table 2. Compared with the healthy control group, the $\delta$ relative band power in the posterior area was significantly increased $(p$ $=0.024)$ in the anti-NMDAR patient group, while $\beta$ relative $(p$ $=0.006)$ and $\beta$ absolute $(p=0.008)$ band power in the posterior area were significantly reduced. The qEEG parameters in anterior area showed no significant differences between the patient group and the control group. There were also no significant differences in qEEG parameters between critically ill and non-critically ill subgroups, or between patients with favorable and poor shortterm outcomes. However, in terms of long-term outcomes, the aEEG bandwidth in the parietal area was significantly lower in patients with favorable outcomes than those with poor outcomes (1.6 vs. $1.95 \mu \mathrm{V}, p=0.030$ ). The parietal total power was also significantly lower in the favorable long-term outcome subgroup compared with the poor outcome subgroup (10.68 vs. $27.9 \mu \mathrm{V}^{2}$, $p=0.022$ ).

We also investigated whether the differences in qEEG parameters between anterior and posterior areas were correlated with severities or outcomes (see Table 3). In the healthy control group, we found that the aEEG lower margin, $\delta$ relative band power, $\alpha$ relative band power, and $\alpha$ absolute band power differed statistically between the anterior and posterior areas. The aEEG lower margin, $\alpha$ relative band power, and $\alpha$ absolute power exhibited a decline from anterior to posterior areas, while $\delta$ relative band power showed the opposite trend. However, in the patient group, the differences in aEEG lower margin, $\delta$ relative band power, and $\alpha$ absolute power vanished. The gradient of $\alpha$ relative band power still remained, and new gradients of $\delta$ absolute band power and $\beta$ absolute band power appeared in the patient group. The gradients of $\alpha$ relative band power, $\delta$ absolute band power and $\beta$ absolute band power existed in the noncritically ill subgroup, whereas the differences in all parameters disappeared in critically ill subgroup.

Taking the long-term outcome as dependent variable, with univariate logistic regression we screened parietal aEEG upper margin, aEEG bandwidth, SEF-95, and $\beta$ relative band power as independent variables. Subsequent multivariate logistic regression analysis yielded only one predictor: the parietal aEEG bandwidth (odds ratio, 37.9; 95\% confidence interval, 1.11$1295.27 ; p=0.044)$. The maximal index of Youden was 1.4625 for a cutoff value of $1.7 \mu \mathrm{V}$, with a sensitivity and specificity of $90.00 \%$ and $56.25 \%$, respectively. ROC analysis of parietal aEEG bandwidth yielded an area under the curve of 0.7312 (95\% CI: 0.572-0.891; Figure 1). Furthermore, using another univariate logistic regression analysis, we found that parietal aEEG bandwidth was associated with long-term moderate/severe disability (mRS score $\geq 3$ ) at 12 months (odds ratio, 761.88; 95\% confidence interval, $1.53-378836.40 ; p=0.036)$, but not associated with relapse events $(p=0.611)$.

\section{DISCUSSION}

Anti-NMDAR encephalitis has been recognized as a common cause of encephalitis in ICU. Despite its responsiveness to

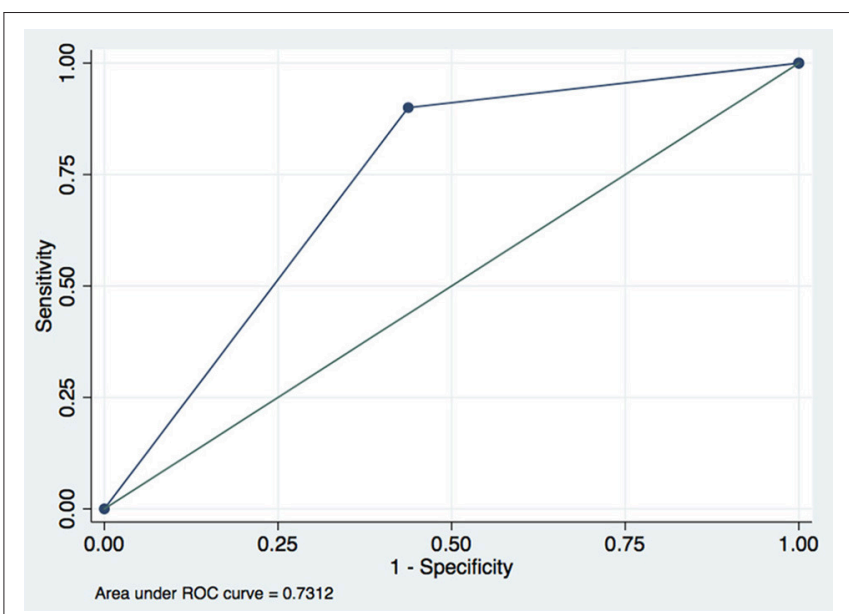

FIGURE 1 | Receiver operating characteristic curve of parietal aEEG bandwidth predicting long-term outcomes of anti-NMDAR encephalitis when cutoff point is 1.7 .

immunotherapy and tumor removal, the mortality rate of antiNMDAR encephalitis in the ICU is $4-25 \%(4,13-15)$. At present, there is no reliable tool for predicting outcomes of antiNMDAR encephalitis. Most previous electrophysiological studies focused on raw EEG manifestations of anti-NMDAR encephalitis $(6,8,9)$. In this study, we investigated the characteristics of qEEG in patients with anti-NMDAR encephalitis. To the best of our knowledge, this is the first study that depicts qEEG findings of patients with anti-NMDAR encephalitis. Our results indicate that most qEEG parameters, including aEEG background, total power, SEF-95, and power of various frequency bands of brain rhythms failed to measure the clinical severity of anti-NMDAR encephalitis. However, the widening of parietal aEEG bandwidth can be used as an objective marker to predict poor long-term outcomes with a good sensitivity.

aEEG is a type of processed EEG that is compressed with respect to amplitude and time, and the upper and lower margins of the aEEG reflect the maximum/minimum peakto-peak amplitudes of the EEG signals (16). An abnormal aEEG, especially its lower margins, has been shown to be predictive of persistence of severe cerebral injury and poor neurologic outcome (17-19). However, aEEG bandwidth is not a commonly used monitoring marker. The prognostic value of parietal aEEG bandwidth in anti-NMDAR encephalitis is a novel finding and difficult to explain. Previous studies on aEEG have mainly concentrated on disorders that lead to neuron damage, such as stroke, traumatic brain injury, or hypoxic encephalopathy, which can be appraised by aEEG lower margins. However, anti-NMDAR encephalitis selectively reduces NMDAR function and changes the synaptic activities of neuronal networks without any impairment of other synaptic processes (20). Aberrant functioning of a single ion channel may result in various pathophysiological processes (20); therefore, the degree of NMDAR hypofunction may not have a linear 


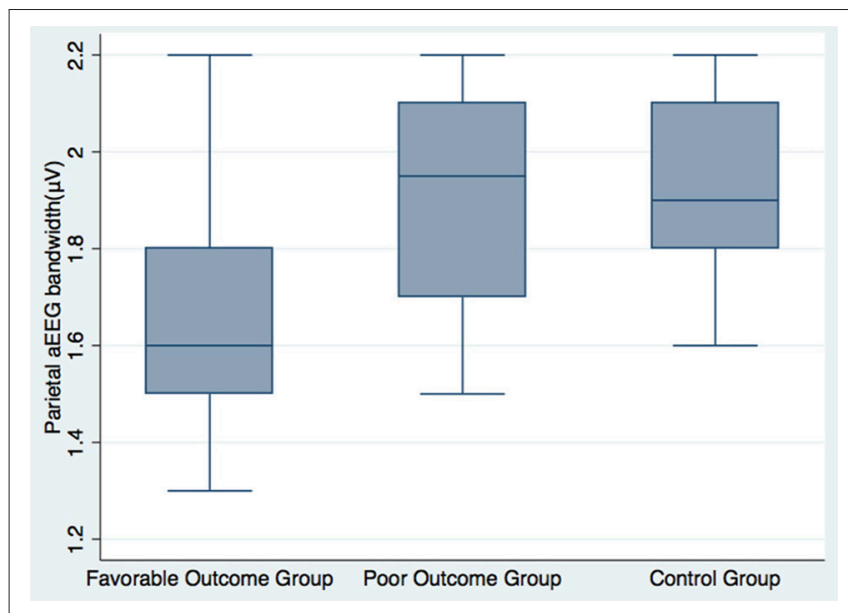

FIGURE 2 | Parietal aEEG bandwidth among favorable long-term patients, poor long-term patients, and healthy volunteers.

relationship with EEG discontinuity. Thus, the subsequent aEEG changes may be difficult to evaluate using traditional aEEG lower margin. On the contrary, unlike simply measuring the lower margin, aEEG bandwidth may play a diagnostic and predictive role in disorders that are pathogenic to synapses. Specifically, the parietal aEEG bandwidth in healthy controls was wider than in patients with favorable long-term outcomes, and narrower than in those with poor long-term outcomes (Figure 2). This opposite trend suggests that the long-term outcome of anti-NMDAR encephalitis may be determined by underlying, unexplained pathophysiological mechanisms, which might be reflected in electrophysiological parietal aEEG bandwidth. The molecular mechanism underlying this requires further investigation.

Interestingly, compared with healthy controls, the lower margins of the aEEG in both the critically ill and non-critically ill subgroup did not show any significant differences. One possible reason for this phenomenon is the small number of patients. Nevertheless, it may also suggest that brain function in antiNMDAR encephalitis is relatively intact even in critically ill patients. This characteristic of qEEG is potentially consistent with the pathogenesis of anti-NMDAR encephalitis. NMDAR is an ionotropic glutamate receptor distributed in entire brain tissues. Antibodies directed at the NR1 subunit of the NMDA receptors act by mechanisms including the binding, capping, and cross-linking of NMDA receptors, leading to internalization from the cell membrane surface and a selective decrease in NMDA receptor currents with no effect on synapse number or other synapse proteins $(2,21)$. The NMDAR hypofunction is non-destructive and reversible, which may explain why the lower margins of aEEG did not decline in the patient group.

Compared with the healthy control group, there were specific anterior-to-posterior graded alterations of qEEG parameters in patients with anti-NMDAR encephalitis. In particular, there were alterations in $\delta$ and $\beta$ absolute band power. $\delta$ absolute band power in the posterior area was lower than in the anterior area in the healthy control group and non-critically ill subgroup, but higher than in anterior area of the critically ill subgroup. However, this trend was reversed in the $\beta$ absolute band power, which was higher in the posterior area in the healthy control group, and lower in the critically ill and non-critically ill subgroups. Increased power in slower frequency bands ( $\delta$ and $\theta)$ and decreased power in faster frequency bands $(\alpha$ and $\beta$ ) are seen with reductions in brain metabolism (22). No previous studies have reported anterior-to-posterior gradient changes in electrophysiology in anti-NMDAR encephalitis; however, several FDG-PET/CT-based studies have observed specific anterior-to-posterior metabolic gradient changes in the active phase of NMDA encephalitis, and reported these to correlate with disease severity and renormalize with treatment and recovery (23-25). The observed anterior-to-posterior gradient may largely be driven by posterior hypometabolism rather than anterior hypermetabolism (25). Wegener et al. have identified a predominant pattern of frontotemporal hypermetabolism and parietal hypometabolism (26). However, they found that there were no consistent results regarding FD-PET results and impairment as indicated by the mRS (26), which is consistent with our study relative to the prognostic value of the anterior-to-posterior gradient. Another FDG-PET/CTbased study demonstrated marked posterior hypometabolism in patients with anti-NMDAR encephalitis with severe neurologic disability (mRS 4-5), which was more evident than in those less neurologically disabled (mRS 0-3) (25). In our study, the posterior $\delta$ absolute band power in non-critically ill subgroup is lower than the anterior area, and this discrepancy disappeared in the critically ill subgroup, which may indicate that more a significant posterior hypometabolism emerged in the critically ill subgroup and supports the research of Probasco et al.

The main limitation of our study is the small number of patients, which limits the power of the findings. In addition, patients requiring qEEG monitoring due to decreased consciousness or suspected seizures, but were not severe enough to require ICU admission, were enrolled as the non-critically ill subgroup. This might have led to a selection bias; however, it also enabled the analysis of the most challenging group of patients with this disease, in whom prognostic biomarkers are most needed. Additional analysis of temporal and occipital areas, as well as prolonged qEEG monitoring, are needed in the future. Furthermore, while critically ill patients are monitored, some were being administered with anti-epileptic drugs, sedatives, or antipsychotics at the same time. In our study, almost all patients in the ICU was administered at least one intravenous sedative, including midazolam, diazepam, or propofol, to control seizures and involuntary movements in the early course of the disease. Before the start of qEEG monitoring, we requested that these sedatives be suspended and restarted after the monitoring is over. However, these medications may still have an impact on EEG signals. In general, sedatives and antiepileptic medications depress the electrocortical activity and render the EEG background more discontinuous and depressed than expected; therefore, a continuous background may become slightly discontinuous (27). If the EEG background is considered normal there is typically no problem with interpretation. In this 
study, the aEEG lower margins of all patients were continuous $(>5 \mu \mathrm{V})$; therefore, sedatives and anti-epileptic medications were unlikely to have had a significant impact on our EEG results.

In conclusion, the qEEG pattern in anti-NMDAR encephalitis can offer better understanding of the pathophysiological mechanisms and prognostic possibilities. A wider parietal aEEG bandwidth was associated with worse long-term outcomes, and may serve as a useful biomarker in anti-NMDAR encephalitis. Further, well-designed studies are needed to confirm this novel finding, and elucidate the underlying mechanism.

\section{AUTHOR CONTRIBUTIONS}

NJ wrote the initial draft of the paper. HG and HR acquisitioned patients' demographic and clinical data from encephalitis database registration. QL guided for analyzing the EEG signals.

\section{REFERENCES}

1. Dalmau J, Gleichman AJ, Hughes EG, Rossi JE, Peng X, Lai M, et al. Anti-NMDA-receptor encephalitis: case series and analysis of the effects of antibodies. Lancet Neurol. (2008) 7:1091-8. doi: 10.1016/S1474-4422(08)70224-2

2. Hughes EG, Peng X, Gleichman AJ, Lai M, Zhou L, Tsou R, et al. Cellular and synaptic mechanisms of anti-NMDA receptor encephalitis. J Neurosci. (2010) 30:5866-75. doi: 10.1523/JNEUROSCI.016710.2010

3. Gable MS, Sheriff H, Dalmau J, Tilley DH, Glaser CA. The frequency of autoimmune $\mathrm{N}$-methyl-D-aspartate receptor encephalitis surpasses that of individual viral etiologies in young individuals enrolled in the California Encephalitis Project. Clin Infect Dis. (2012) 54:899-904. doi: $10.1093 / \mathrm{cid} / \operatorname{cir} 1038$

4. Chi X, Wang W, Huang C, Wu M, Zhang L, Li J, et al. Risk factors for mortality in patients with anti-NMDA receptor encephalitis. Acta Neurol Scand. (2016) 136:298-304. doi: 10.1111/ane.12723

5. DeSena $\mathrm{AD}$, Greenberg $\mathrm{BM}$, Graves D. Three phenotypes of anti$\mathrm{N}$-methyl-D-aspartate receptor antibody encephalitis in children: prevalence of symptoms and prognosis. Pediatr Neurol. (2014) 51:542-9. doi: 10.1016/j.pediatrneurol.2014.04.030

6. Schmitt SE, Pargeon K, Frechette ES, Hirsch LJ, Dalmau J, Friedman D, et al. Extreme delta brush: a unique EEG pattern in adults with anti-NMDA receptor encephalitis. Neurology (2012) 79:1094-100. doi: 10.1212/WNL.0b013e3182698cd8

7. Foff EP, Taplinger D, Suski J, Lopes MB, Quigg M. EEG findings may serve as a potential biomarker for anti-NMDA receptor encephalitis. Clin EEG Neurosci. (2016) 48:48-53. doi: 10.1177/1550059416642660

8. Zhang Y, Liu G, Jiang MD, Li LP, Su YY. et al. Analysis of electroencephalogram characteristics of anti-NMDA receptor encephalitis patients in China. Clin Neurophysiol. (2017) 128:1227-1233. doi: 10.1016/j.clinph.2017.04.015

9. Limotai C, Denlertchaikul C, Saraya AW, Jirasakuldej S.. Predictive values and specificity of electroencephalographic findings in autoimmune encephalitis diagnosis. Epilepsy Behav. (2018) 84:29-36. doi: 10.1016/j.yebeh. 2018.04.007

10. Wu Y, Chen M, Cui Y, He X, Niu J, Zhang Y, et al. Viral encephalitis in quantitative EEG. J Integr Neurosci. (2018) 17:493-501. doi: $10.3233 /$ jin-180084

11. Foreman B, Claassen J. Quantitative EEG for the detection of brain ischemia. Crit Care (2012) 16:216. doi: 10.1186/cc11230

12. Graus F, Titulaer MJ, Balu R, Benseler S, Bien CG, Cellucci T, et al. A clinical approach to diagnosis of autoimmune encephalitis. Lancet Neurol. (2016) 15:391-404. doi: 10.1016/S1474-4422(15)00401-9
BP guided for study designation and made critical revision of draft.

\section{FUNDING}

Exploration on the Training Mode of Postgraduate Specialists in Neurology (Item Number 10023201600104).

\section{ACKNOWLEDGMENTS}

We gratefully acknowledge the support from Huadong Zhu (Emergency Intensive Care Unit, Peking Union Medical College Hospital, Chinese Academy of Medical Sciences, Beijing, P. R. China) and Bin Du (Medical Intensive Care Unit, Peking Union Medical College Hospital, Chinese Academy of Medical Sciences, Beijing, P. R. China) for their support of this research.

13. Chen X, Li J-M, Liu F, Wang Q, Zhou D, Lai X. Anti-N-methyl-d-aspartate receptor encephalitis: a common cause of encephalitis in the intensive care unit. Neurological Sci. (2016) 37:1993-8. doi: 10.1007/s10072-0162702-y

14. de Montmollin E, Demeret S, Brule N, Conrad M, Dailler F, Lerolle N, et al. Anti-N-methyl-D-aspartate receptor encephalitis in adult patients requiring intensive care. Am J Respir Crit Care Med. (2016) 195:491-9. doi: 10.1164/rccm.201603-0507OC

15. Harutyunyan G, Hauer L, Dunser MW, et al. Autoimmune encephalitis at the neurological intensive care unit: etiologies, reasons for admission and survival. Neurocrit Care (2016) 27:82-9. doi: 10.1007/s12028-016-0370-7

16. Zhang D, Ding H. Calculation of compact amplitude-integrated EEG tracing and upper and lower margins using raw EEG data. Health (2013) 05:885-891. doi: $10.4236 /$ health.2013.55116

17. Hellstrom-Westas L, Rosen I, Svenningsen NW. Predictive value of early continuous amplitude integrated EEG recordings on outcome after severe birth asphyxia in full term infants. Arch Dis Child Fetal Neonatal Ed. (1995) 72:F34-8.

18. Toet MC, Hellstrom-Westas L, Groenendaal F, Eken P, de Vries LS. Amplitude integrated EEG 3 and 6 hours after birth in full term neonates with hypoxic-ischaemic encephalopathy. Arch Dis Child Fetal Neonatal Ed. (1999) 81:F19-23.

19. al Naqeeb N, Edwards AD, Cowan FM, Azzopardi D. Assessment of neonatal encephalopathy by amplitude-integrated electroencephalography. Pediatrics (1999) 103(6 Pt 1):1263-71.

20. Irani SR, Vincent A. NMDA receptor antibody encephalitis. Curr Neurol Neurosci Rep. (2011) 11:298-304. doi: 10.1007/s11910-011-0186-y

21. Dalmau J, Lancaster E, Martinez-Hernandez E, Rosenfeld MR, BaliceGordon R. Clinical experience and laboratory investigations in patients with anti-NMDAR encephalitis. Lancet Neurol. (2011) 10:63-74. doi: 10.1016/s1474-4422(10)70253-2

22. Nagata K, Tagawa K, Hiroi S, Shishido F, Uemura K. Electroencephalographic correlates of blood flow and oxygen metabolism provided by positron emission tomography in patients with cerebral infarction. Electroencephalogr Clin Neurophysiol. (1989) 72:16-30.

23. Leypoldt F, Buchert R, Kleiter I, Marienhagen J, Gelderblom M, Magnus T, et al. Fluorodeoxyglucose positron emission tomography in anti-N-methyl-Daspartate receptor encephalitis: distinct pattern of disease. J Neurol Neurosurg Psychiatry (2012) 83:681-6. doi: 10.1136/jnnp-2011-301969

24. Yuan J, Guan H, Zhou X, Niu N, Li F, Cui L, Cui R. Changing brain metabolism patterns in patients with ANMDARE: serial 18F-FDG PET/CT findings. Clin Nucl Med. (2016) 41:366-70. doi: 10.1097/RLU.0000000000001164

25. Probasco JC, Solnes L, Nalluri A, Cohen J, Jones KM, Zan E, et al. Decreased occipital lobe metabolism by FDG-PET/CT: an anti-NMDA receptor 
encephalitis biomarker. Neurol Neuroimmunol Neuroinflamm. (2018) 5:e413. doi: 10.1212/NXI.0000000000000413

26. Wegner F, Wilke F, Raab P, Tayeb SB, Boeck AL, Haense C, et al. Anti-leucine rich glioma inactivated 1 protein and anti-N-methylD-aspartate receptor encephalitis show distinct patterns of brain glucose metabolism in 18F-fluoro-2-deoxy-d-glucose positron emission tomography. BMC Neurol. (2014) 14:136. doi: 10.1186/1471-237714-136

27. Hellströmwestas L, Vries LSD, Rosen I. An Atlas of Amplitude-Integrated EEGs in the Newborn, 2nd Edn. London: Encyclopedia of Visual Medicine Series (2008).
Conflict of Interest Statement: The authors declare that the research was conducted in the absence of any commercial or financial relationships that could be construed as a potential conflict of interest.

Copyright (c) 2018 Jiang, Guan, Lu, Ren and Peng. This is an open-access article distributed under the terms of the Creative Commons Attribution License (CC BY). The use, distribution or reproduction in other forums is permitted, provided the original author(s) and the copyright owner(s) are credited and that the original publication in this journal is cited, in accordance with accepted academic practice. No use, distribution or reproduction is permitted which does not comply with these terms. 